\title{
Effect of Growth Rate on Streptomycin Accumulation by Escherichia coli and Bacillus megaterium
}

\author{
By MARIANNE E. MUIR, ROBYN S. VAN HEESWYCK† \\ AND BRIAN J. WALLACE* \\ School of Microbiology, Faculty of Biological Sciences, The University of New South Wales, \\ Kensington, NSW 2033, Australia
}

(Received 5 September 1983; revised 20 March 1984)

\begin{abstract}
The rate of accumulation of streptomycin by streptomycin-sensitive strains of Escherichia coli and Bacillus megaterium, grown in chemostats, was related to the growth rate prior to addition of the antibiotic. For $E$. coli the length of the lag period that preceded accumulation was also growth rate-dependent. Thus faster growing cultures accumulated streptomycin more rapidly and with a shorter lag than slower growing cultures. The rate of efflux of streptomycin from bacteria that had accumulated streptomycin was not greatly influenced by growth rates of the cultures. At a particular growth rate, accumulation of streptomycin was found to be faster at higher concentrations of the antibiotic. Rapid accumulation of streptomycin was not observed with continuous cultures of a streptomycin-resistant strain of $E$. coli. Accumulation of streptomycin was abolished when growth was inhibited by either terminating the flow of fresh medium to a chemostat or by adding inhibitors that block protein synthesis. These results suggest that the rate of accumulation of streptomycin is related to the concentration of streptomycin-sensitive ribosomes that are actively engaged in protein synthesis within the bacterial cells.
\end{abstract}

\section{INTRODUCTION}

It has been known for many years that streptomycin does not cause loss of viability of Escherichia coli or Staphylococcus aureus under conditions where growth of the organisms cannot occur (Anand \& Davis, 1960; Hancock, 1960). Kogut \& Harris (1969) showed that the growthinhibitory effects of streptomycin and dihydrostreptomycin were influenced by the growth rate of the culture prior to antibiotic addition. Thus, the faster the growth rate of a bacterial culture, the greater was the inhibition of growth by streptomycin. Kogut \& Harris (1969) were unable to account for this result by an effect of growth rate on intracellular accumulation of streptomycin. Instead, they explained their observation in terms of an increased availability of ribosomal targets for streptomycin (due to increased ribosome synthesis rates at faster growth rates). However, in their streptomycin accumulation experiments, they used $\left[{ }^{14} \mathrm{C}\right]$ streptomycin of low specific activity. The low intracellular counts that resulted made it difficult to assess adequately the effects of growth rates on streptomycin accumulation. For this reason we re-examined streptomycin accumulation by bacterial cultures grown at controlled rates in small chemostats. Our results show that the rate of streptomycin accumulation by cultures of $E$. coli and Bacillus megaterium is dependent on the growth rate of the bacterial culture prior to aminoglycoside addition.

† Present address: Carlsberg Laboratory, Department of Physiology, GL Carisberg VEJ10, DK-2500, Copenhagen, Valby, Denmark.

Abbreviation: AUM, aminoglycoside uptake medium. 


\section{METHODS}

Bacterial strains. Bacillus megaterium (strain ATCC 0011200) was obtained from our Faculty culture collection. All other strains used were derivatives of E. coli K12. JP2140 [ilv-1, his-29(am), trpA9605(am)] was obtained from A. J. Pittard, School of Microbiology, University of Melbourne, Victoria, Australia. NSW5 is an $i l v^{+}$transductant derived from JP2140 and NSW3 is a spontaneous streptomycin-resistant (rps $L$ ) derivative of JP2140. Genetic nomenclature used conforms to that recommended by Demerec et al. (1968), and the symbols for gene loci are those of Bachmann \& Low (1980).

Media and growth of bacteria. Cultures were maintained on solid medium consisting of nutrient broth (Oxoid) enriched with $0.5 \%(\mathrm{w} / \mathrm{v})$ brain heart infusion (Oxoid) and supplemented with $2 \%(\mathrm{w} / \mathrm{v})$ agar (Difco). In all experiments growth was at $37^{\circ} \mathrm{C}$. For batch culture experiments bacteria were grown in 10 or $20 \mathrm{ml}$ aminoglycoside uptake medium (AUM; Bryan \& Van Den Elzen, 1977) in $125 \mathrm{ml}$ shake flasks. AUM was supplemented with one of the following carbon sources at a final concentration of $10 \mathrm{mM}$ : glucose, mannitol, galactose or succinate (to provide generation times of $0.83 \mathrm{~h}, 0.83 \mathrm{~h}, 2.8 \mathrm{~h}$ and $8.3 \mathrm{~h}$, respectively). The following additions were made as required: thiamin. $\mathrm{HCl}(3 \mu \mathrm{M})$, L-isoleucine $(0.38 \mathrm{mM})$, L-valine $(0.43 \mathrm{~mm})$, L-histidine $(0.52 \mathrm{~mm})$, and L-tryptophan ( $0.2 \mathrm{~mm})$. Generation times (doubling times) for strains growing in batch culture were calculated from the slope of the linear (exponential) portion of plots of log OD against time. For convenience we will refer to the growth rate of the bacterial culture prior to streptomycin addition as the initial growth rate. For chemostat cultures the initial growth rate is also the dilution rate under steady-state conditions.

For continuous culture experiments, bacteria were grown in a chemostat with a working volume of $20 \mathrm{ml}$. The chemostat consisted of a stoppered wide-bore glass tube, with two ports, one allowing inlet of medium and air and the other for sample withdrawal. The vessel was maintained at $37^{\circ} \mathrm{C}$ with a circulating water bath. An outlet tube permitted escape of spent culture fluid and gas. The chemostat was mixed and aerated by bubbling sterile instrument grade air (CIG, Australia) at $20 \mathrm{ml} \mathrm{min}^{-1}$ through a sintered glass sparger located at the base of the culture vessel. The sparger also permitted entry of fresh growth medium. Medium was pumped into the chemostat by a peristaltic pump (LKB Microperpex Model 2132). For growth in the chemostat, AUM was used for $E$. coli strains and Medium A (Davis \& Mingioli, 1950) for B. megaterium. Glucose was present at $1 \mathrm{~mm}$. Casamino acids (Difco, vitamin-free) were added to $E$. coli cultures at $0.1 \%(w / v)$ and at $0.025 \%(w / v)$ for $B$. megaterium. Additional supplements (listed above) were added as required. Under these conditions growth was limited by the available carbon source since increasing the concentration of glucose or Casamino acids resulted in proportionately higher cell densities. The $\mathrm{pH}$ of chemostat cultures growing in steady-state at dilution rates of $0.71 \mathrm{~h}^{-1}$ and $0.19 \mathrm{~h}^{-1}$ was the same as that of the uninoculated medium. Growth was monitored throughout by OD measurements in a Klett-Summerson colorimeter fitted with a blue filter. Cultures of both $E$. coli and $B$. megaterium with ODs equivalent to $100 \mathrm{Klett}$ units were found to contain $0.14 \mathrm{mg}$ dry weight of bacteria $\mathrm{ml}^{-1}$. For measurements of growth in the chemostat, samples $(5 \mathrm{ml})$ were removed aseptically with a sterile syringe fitted with a $15 \mathrm{~cm}$ needle, and returned as soon as the $O D$ had been determined in a sterile cuvette. This procedure did not affect steady-state growth or cause culture contamination. The purity of chemostat cultures was checked routinely by plating onto solid media.

Streptomycin accumulation experiments. Accumulation experiments in batch cultures were carried out as previously described (Muir \& Wallace, 1979) using streptomycin at $10 \mu \mathrm{g}(0.77 \mu \mathrm{Ci} ; 28.5 \mathrm{kBq}) \mathrm{ml}^{-1}$ final concentration. For chemostat cultures, uptake experiments were started when steady-state growth had been attained, usually after the passage of at least five vessel volumes. A mixture of streptomycin and $\left[{ }^{3} \mathrm{H}\right]$ dihydrostreptomycin was added first to the medium supply and then, at the same final concentration, to the chemostat at a time coincident with the arrival at the culture of the medium containing labelled streptomycin (zero time). In this way, bacteria in the chemostat were exposed continuously from zero time to the same final concentration of streptomycin. Generally a final streptomycin concentration of $20 \mu \mathrm{g}(1.54 \mu \mathrm{Ci}) \mathrm{ml}^{-1}$ was used. In some experiments higher or lower streptomycin concentrations were used; the specific activity however remained the same, except for experiments with B. megaterium where the concentration of streptomycin was $2 \mu \mathrm{g}(0 \cdot 77 \mu \mathrm{Ci})$ $\mathrm{ml}^{-1}$ and for experiments where streptomycin was used at $150 \mu \mathrm{g}(0.51 \mu \mathrm{Ci}) \mathrm{ml}^{-1}$. Two $100 \mu \mathrm{l}$ samples were removed from the chemostat at zero time and at appropriate time intervals thereafter, and processed for radioactive counting as described previously (Muir \& Wallace, 1979). The OD of the culture was monitored at intervals throughout the experiment so that the radioactivity present in each sample could be related to dry weight of bacteria present. Rates of streptomycin accumulation were calculated from the gradient of the linear part of the curve in which accumulated streptomycin was plotted against time.

Streptomycin efflux experiments. Cultures of strain JP2140 were grown in chemostats at dilution rates of $0.68 \mathrm{~h}^{-1}$ and $0.19 \mathrm{~h}^{-1}$. Streptomycin at $20 \mu \mathrm{g}(1.54 \mu \mathrm{Ci}) \mathrm{ml}^{-1}$ was added to the chemostats and media inlet flasks and incubated for $45 \mathrm{~min}$ and $150 \mathrm{~min}$, respectively, for cultures at dilution rates of $0.68 \mathrm{~h}^{-1}$ and $0 \cdot 19 \mathrm{~h}^{-1}$. The bacteria were then collected by centrifugation at $2{ }^{\circ} \mathrm{C}$, washed once with ice-cold AUM and resuspended to the original cell density in fresh medium prewarmed to $37^{\circ} \mathrm{C}$. Samples $(0.5 \mathrm{ml})$ were removed at regular intervals, filtered and counted to determine the radioactivity remaining associated with the bacteria. 


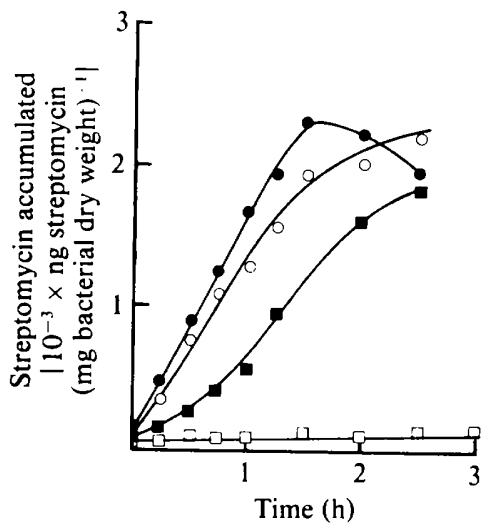

Fig. 1. Effect of growth rate on accumulation of streptomycin by batch cultures of $E$. coli. Strain JP2140 was grown to early exponential phase in AUM $(20 \mathrm{ml})$ supplemented with $10 \mathrm{mM}$-mannitol $(\odot)$, glucose $(O)$, galactose $(\square)$ or succinate $(\square)$. When a dry weight of $0.084 \mathrm{mg} \mathrm{ml}^{-1}$ had been reached, streptomycin $\left.\left[10 \mu \mathrm{g}(0.77 \mu \mathrm{Ci}) \mathrm{ml}^{-1}\right)\right]$ was added. At various times thereafter $0.5 \mathrm{ml}$ samples were removed and the radioactive label present in the bacteria was determined as described in Methods. Generation times were: mannitol, $0.83 \mathrm{~h}$; glucose, $0.83 \mathrm{~h}$; galactose, $2.8 \mathrm{~h}$; succinate, $8.3 \mathrm{~h}$.

\section{RESULTS}

Effect of growth rate on streptomycin accumulation in batch culture

Initially we investigated the influence of growth rate on streptomycin accumulation by $E$. coli K12 (strain JP2140) growing in batch cultures supplemented with different carbon sources to provide generation times in the range $0.83 \mathrm{~h}$ to $8.3 \mathrm{~h}$. When cultures reached early exponential phase, labelled streptomycin $\left(10 \mu \mathrm{g} \mathrm{ml}^{-1}\right)$, was added and accumulation experiments were performed. In cultures supplemented with carbon sources other than glucose, the rate of streptomycin accumulation was related to the initial growth rate in each culture (Fig. 1). Thus, the culture containing mannitol (generation time, $0.83 \mathrm{~h}$ ) showed the fastest accumulation, while that containing succinate (generation time, $8.3 \mathrm{~h}$ ) had the slowest accumulation. Accumulation was less rapid in the culture supplemented with glucose than in the mannitolsupplemented culture although both cultures had the same generation time. Although this result suggested that the rate of streptomycin accumulation was dependent on the initial growth rate of the culture, supplementation of batch cultures with various carbon sources could have generated cells with different physiological properties which might then have influenced the accumulation of streptomycin. Thus Höltje (1978) reported that supplementation with glucose reduced the rate of streptomycin accumulation and suggested that the latter process was under cyclic AMP control.

\section{Effect of dilution rate on streptomycin accumulation in continuous culture}

Figure 2 shows the kinetics of streptomycin $\left(20 \mu \mathrm{g} \mathrm{ml}^{-1}\right)$ accumulation by chemostat cultures of E. coli strain JP2140 at dilution rates in the range 0.67 to $0.09 \mathrm{~h}^{-1}$. Both the duration of the lag phase prior to the onset of rapid accumulation, and the final rate of accumulation of streptomycin varied with the initial growth rate of the culture. Thus, at the highest dilution rate $\left(0.67 \mathrm{~h}^{-1}\right)$ a short lag period of about $10 \mathrm{~min}$ preceded rapid accumulation. At a $7 \cdot 4$-fold lower dilution rate $\left(0.09 \mathrm{~h}^{-1}\right)$ the lag phase lasted 3.5 to $4 \mathrm{~h}$ and accumulation was much slower than in the faster growing cultures. Essentially the same results were obtained when growth of strain JP2140 (a tryptophan auxotroph) was limited by L-tryptophan rather than by carbon source (M. Muir, unpublished results). 


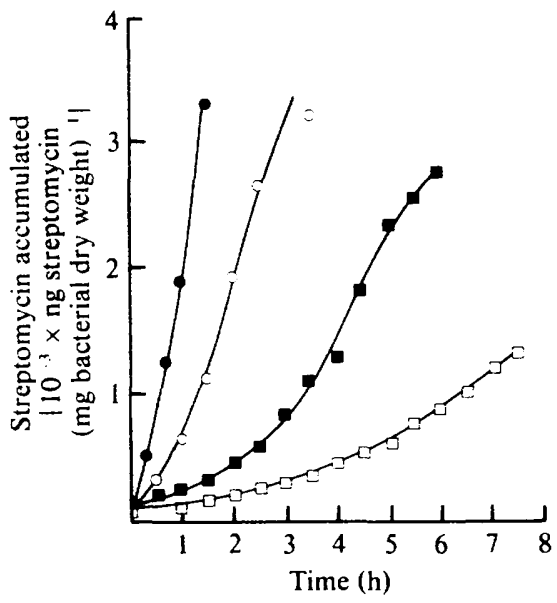

Fig. 2

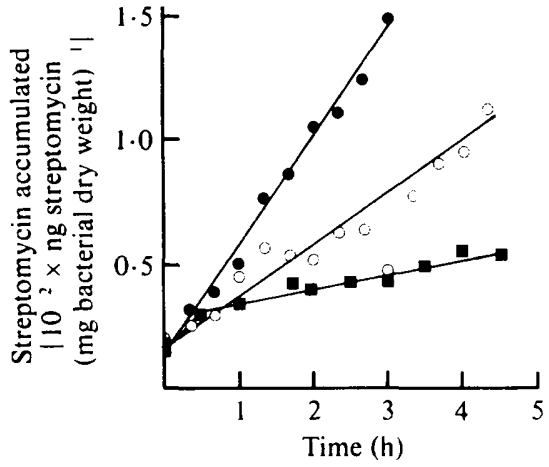

Fig. 3

Fig. 2. Effect of dilution rate on accumulation of streptomycin by $E$. coli in continuous culture. Strain JP2140 was grown in a small chemostat in AUM with glucose $(1 \mathrm{mM})$ and Casamino acids $(0.1 \%$, w/v $)$ in addition to the other required supplements (see Methods). When steady-state growth was reached. streptomycin $\left[20 \mu \mathrm{g}(1.54 \mu \mathrm{Ci}) \mathrm{ml}^{-1}\right]$ was added to the medium reservoir and the chemostat such that the concentration of the antibiotic remained at $20 \mu \mathrm{g} \mathrm{m}^{-1}$ thereafter (zero time). Duplicate $100 \mu \mathrm{l}$ samples of culture were removed at suitable intervals and the radioactivity present in the cells was determined as described in Methods. Dilution rates: $0.67 \mathrm{~h}^{-1}(O) ; 0.37 \mathrm{~h}^{-1}(O) ; 0.17 \mathrm{~h}^{-1}(\square)$; $0.09 \mathrm{~h}^{-1}(\square)$.

Fig. 3. Effect of dilution rate on streptomycin accumulation by $B$. megaterium in continuous culture. Strain ATCC 0011200 was grown in a small chemostat in Medium A with glucose (1 mM) and Casamino acids $(0.025 \%, \mathrm{w} / \mathrm{v})$. When steady-state growth was reached, streptomycin $[2 \mu \mathrm{g}(0.77 \mu \mathrm{Ci})$ $\mathrm{ml}^{-1}$ ] was added and accumulation was measured as in Fig. 2. Dilution rates: $0.68 \mathrm{~h}^{-1}(0) ; 0.34 \mathrm{~h}^{-1}$ (O); $0 \cdot 19 \mathrm{~h}^{-1}$ (D).

\section{Effect of dilution rate on efflux of streptomycin}

The accumulated streptomycin was slowly lost when bacteria that had been incubated with streptomycin were incubated in a streptomycin-free medium. The rate of efflux depended on the initial growth rate of the culture, but the difference in efflux rates between two cultures grown at different rates was relatively small. Thus a culture grown at a dilution rate of $0.68 \mathrm{~h}^{-1}$ and then loaded with streptomycin (see Methods), retained $80 \%$ of the streptomycin after $60 \mathrm{~min}$ incubation in a streptomycin-free medium. A culture grown at a dilution rate of $0.19 \mathrm{~h}^{-1}$ retained $90 \%$ of the streptomycin over the same period. It can be concluded that the low rates of streptomycin accumulation observed in slow growing cultures were not due to rapid efflux of the drug.

\section{Effect of dilution rate on streptomycin accumulation by B. megaterium in continuous culture}

Since the dependence of accumulation rates on growth rate could have been restricted to Gram-negative organisms (due perhaps to an effect mediated by the presence of an outer membrane), we examined accumulation of streptomycin by a Gram-positive organism, $B$. megaterium. As this organism is known to be highly sensitive to aminoglycosides (Hancock, $1962 a)$ a lower final streptomycin concentration $\left(2 \mu \mathrm{g} \mathrm{ml}^{-1}\right)$ and a higher specific activity were used for these experiments. As for $E$. coli, accumulation rates for $B$. megaterium were found to be related to the dilution rates of the cultures (Fig. 3). In contrast to $E$. coli, however, there was no lag phase prior to the onset of rapid accumulation with $B$. megaterium. The absence of a lag phase was consistent with earlier studies with this organism (Hancock, 1962b) and with its exquisite sensitivity to the antibiotic. However, another Gram-positive organism, Staphylo- 
Table 1. Dependence of rate of accumulation of streptomycin on streptomycin concentration

Strain JP2140 was grown in chemostats at the dilution rates indicated. Accumulation of streptomycin was measured as described in Methods.

$\begin{array}{ccc}\begin{array}{c}\text { Streptomycin concn } \\ \left.(\mu \mathrm{g} \mathrm{m})^{-1}\right)\end{array} & \begin{array}{c}\text { Dilution rate } \\ \left(\mathrm{h}^{-1}\right)\end{array} & \begin{array}{c}\text { Rate* of accumulation } \\ \text { of streptomycin }\end{array} \\ 10 & 0 \cdot 71 & 4 \cdot 5 \\ & 0 \cdot 19 & 1 \cdot 2 \\ 20 & 0 \cdot 79 & 19 \\ & 0 \cdot 19 & 3 \cdot 3 \\ 50 & 0 \cdot 70 & 50 \\ & 0 \cdot 21 & 10 \\ 150 & 0.68 & 225 \\ & 0 \cdot 19 & 20\end{array}$

* Rates are expressed as $\mathrm{ng}$ streptomycin accumulated $\min ^{-1}$ ( $\mathrm{mg}$ bacterial dry weight $)^{-1}$ and were calculated as described in Methods.

coccus aureus, accumulated gentamicin (at 0.5 and $1.0 \mu \mathrm{g} \mathrm{ml}^{-1}$ ) after a short lag phase (Miller et al., 1980).

\section{Effect of growth termination on accumulation of streptomycin in continuous culture}

The effect of sudden reduction in dilution rate on accumulation was examined by terminating medium flow to one chemostat after accumulation had commenced. In this experiment, two chemostats inoculated with $E$. coli strain JP2140 were run at the same dilution rate $\left(0.64 \mathrm{~h}^{-1}\right)$ and, when cultures reached steady-state, accumulation experiments were initiated by addition of streptomycin $\left(20 \mu \mathrm{g} \mathrm{ml}^{-1}\right)$. After $1 \mathrm{~h}$ medium flow to one of the chemostats was terminated, whilst the other was supplied with medium for a further $2 \mathrm{~h}$. Streptomycin accumulation was reduced, compared with the control, as soon as medium flow stopped. Complete cessation of accumulation occurred after a further $40 \mathrm{~min}$. Although the growth rates of cells must already have been reduced (since the cells had been accumulating streptomycin for $1 \mathrm{~h}$ ), a further severe reduction in growth rate, even at this stage, reduced and then abolished accumulation. Continued growth was thus required for accumulation even in bacteria whose growth rates had already been reduced by the presence of streptomycin.

\section{Effect of streptomycin concentration on accumulation at different dilution rates}

The rate of accumulation of streptomycin by batch cultures is known to be influenced by the streptomycin concentration (Bryan \& Van Den Elzen, 1976). For chemostat cultures the rate of accumulation of streptomycin increased as the streptomycin concentration was raised (Table 1). At concentrations of 10 or $20 \mu \mathrm{g} \mathrm{ml}^{-1}$ (i.e. those used for most of the accumulation experiments) it is clear that the accumulation systems for streptomycin in both slow- and fast-growing cultures were not saturated. In most cases the duration of the lag phase became longer as the concentration of streptomycin was lowered. At a low streptomycin concentration (e.g. $10 \mu \mathrm{g}$ $\mathrm{ml}^{-1}$ ), especially with the culture growing at a dilution rate of $0.19 \mathrm{~h}^{-1}$, it was difficult to decide where the lag phase ended and the rapid phase of accumulation began.

\section{Streptomycin accumulation in a ribosomally-resistant mutant in continuous culture}

The rapid phase of streptomycin accumulation is not observed in batch cultures of ribosomally-resistant strains harbouring mutations at the rpsL (strA) locus (Bryan \& Van Den Elzen, 1976). To confirm this result in continuous culture, we grew strain JP2140 and its otherwise isogenic rps $L$ derivative, NSW3, in chemostats at the same dilution rate $\left(0.37 \mathrm{~h}^{-1}\right)$ and followed the accumulation of streptomycin $\left(20 \mu \mathrm{g} \mathrm{ml}^{-1}\right)$ over a $4 \mathrm{~h}$ period. Whereas JP2140 entered a rapid phase of uptake after $1 \mathrm{~h}$ (see Fig. 2), the ribosomally-resistant strain accumulated a negligible amount of streptomycin over a $4 \mathrm{~h}$ period. The rapid phase of accumulation was thus dependent on the presence of streptomycin-sensitive ribosomes. 


\section{Effect of inhibition of protein synthesis on accumulation of streptomycin in batch cultures}

Chloramphenicol, added either prior to or coincident with the addition of streptomycin, inhibits the rapid phase of accumulation of streptomycin (Anand et al., 1960; Hurwitz \& Rosano, 1962). To determine whether or not this effect is specific for chloramphenicol (used here at $\left.100 \mu \mathrm{g} \mathrm{ml}^{-1}\right)$, the effects of puromycin $\left(500 \mu \mathrm{g} \mathrm{ml}^{-1}\right)$, rifampicin $\left(200 \mu \mathrm{g} \mathrm{ml}^{-1}\right)$ and tetracycline $\left(10 \mu \mathrm{g} \mathrm{ml}^{-1}\right)$ on streptomycin accumulation in batch culture were also studied. Unlike the other three compounds which directly inhibit ribosomal function, rifampicin blocks protein synthesis indirectly through its action on DNA-dependent RNA polymerase. At the concentrations chosen, all compounds caused rapid and complete inhibition of protein synthesis in intact cells. In control cultures of strain NSW5, which contained streptomycin $\left(10 \mu \mathrm{g} \mathrm{ml}^{-1}\right)$ alone, slow accumulation occurred for 10 to $15 \mathrm{~min}$, followed by a rapid secondary phase of accumulation. In cultures which contained one of the above inhibitors in addition to streptomycin, slow accumulation of the latter occurred for 15 to $25 \mathrm{~min}$, but the rapid phase of accumulation was absent.

Evidently protein synthesis was required not only for initiation of the rapid phase of streptomycin accumulation but also for its continuation. Thus, when the addition of chloramphenicol to cultures containing streptomycin was delayed until after the rapid phase of accumulation had commenced, further accumulation was inhibited, although not completely abolished. For example, when added $55 \mathrm{~min}$ after streptomycin, chloramphenicol produced approximately $70 \%$ inhibition of streptomycin accumulation. In earlier studies it was reported that chloramphenicol, when added 10 to $20 \mathrm{~min}$ after streptomycin, caused only moderate to poor inhibition of streptomycin accumulation (Höltje, 1978; Hurwitz \& Rosano, 1962; Andry \& Bockrath, 1974). The reason for the discrepancy between these results and our own is not clear.

\section{DISCUSSION}

In this paper we have shown that streptomycin accumulation is dependent on growth rates of bacterial cultures prior to addition of the antibiotic. In cultures of both $E$. coli and $B$. megaterium, the more rapid the initial rate of growth, the faster was the rate of streptomycin accumulation (Figs 1, 2 and 3). In addition to influencing the rate of streptomycin accumulation, the duration of the lag phase prior to the onset of rapid uptake in $E$. coli is also growth rate-dependent : the greater the dilution rate, the shorter the duration of the lag (Fig. 2). Accumulation of streptomycin occurs when the rate of uptake exceeds the rate of efflux. Since the rates of efflux of streptomycin did not differ significantly in cultures that had been grown at two quite different dilution rates, it can be assumed that the effect of growth rate on accumulation reflects an effect mainly on the uptake of streptomycin.

Our observations suggest that the greater growth-inhibitory effects of streptomycin at more rapid growth rates result from a greater rate of uptake of the antibiotic by bacteria that are growing more rapidly. It is generally agreed that at rapid growth rates more active ribosomes are present per cell than in slower growing bacteria (Maaløe \& Kjeldgaard, 1966; Harvey, 1973; Koch, 1980). We propose therefore that the rate of streptomycin uptake is related to the number of streptomycin-sensitive ribosomes actively engaged in protein synthesis. That ribosomal binding is inherently involved in the uptake mechanism is indicated by the lack of rapid accumulation in fully ribosomally-resistant mutants (Bryan \& Van Den Elzen, 1976; see above results), and a reduction in the rate of accumulation in partial ribosomally-resistant mutants (Ahmad et al., 1980).

Since a sudden marked reduction in dilution rate abolished streptomycin accumulation, one might predict that any inhibitor that reduces bacterial growth rate would also reduce aminoglycoside accumulation, irrespective of the mode of action of the inhibitor. This is indeed the case since four inhibitors, chloramphenicol, tetracycline, puromycin and rifampicin, were found to block streptomycin accumulation. Other inhibitors such as potassium cyanide, sodium azide and dinitrophenol, that inhibit streptomycin accumulation (Bryan \& Van Den Elzen, 1976; Andry \& Bockrath, 1974; Höltje, 1978), also cause rapid inhibition of growth (M. Muir, unpublished observations). 
In an earlier paper (Muir et al., 1981) we reported the isolation and characterization of a mutant of $E$. coli that was partially resistant to streptomycin. Streptomycin was accumulated more slowly by the mutant (NSW77) than by the parent strain. Strain NSW77 was found to be mutated at $u b i F$ and to contain 2-octaprenyl-3-methyl-6-methoxy-1,4-benzoquinone in place of ubiquinone in its respiratory membranes. The respiration rate in membranes of NSW77 was considerably less than that of the parent strain and we suggested that this feature was responsible for the reduced accumulation of streptomycin in NSW77 (Muir et al., 1981). However NSW77 grew more slowly in batch culture than the parent strain. In chemostat cultures of NSW77 and the parent strain, where growth rates of the two were identical, the rates of streptomycin accumulation were found to be the same for parent and mutant (M. Muir, unpublished results).

The nature of the uptake system for streptomycin is not yet understood. One suggestion was that streptomycin uptake occurred via an inducible spermidine transport system (Höltje, 1978). Another was that streptomycin formed a complex with an energy-rich component of respiratory membranes (ubiquinone was suggested), which provides energy for uptake (Bryan \& Van Den Elzen, 1977). In this paper we have suggested that the rate of streptomycin uptake is in some way related to the number of active ribosomes in the bacterial cell. Previous studies (Tempest, 1969) have demonstrated that the intracellular concentrations of two ions involved with ribosomal function $\left(\mathrm{Mg}^{2+}\right.$ and $\left.\mathrm{K}^{+}\right)$depend on growth rate. In those studies it was shown that the ratios $\mathrm{Mg}^{2+}:$ RNA and $\mathrm{K}^{+}:$RNA remained constant in cells that were grown at different dilution rates.

The authors wish to acknowledge the skilled technical assistance of Tillie Coster. We also thank A. J. Pittard for strain JP2140 and P. P. Gray for helpful discussions on chemostat design. This project was supported by a grant to B. J. W. from the Australian Research Grants Committee.

\section{REFERENCES}

Ahmad, M. H., ReChenmacher, A. \& Böck, A. (1980). Interaction between aminoglycoside uptake and ribosomal resistance mutations. Antimicrobial Agents and Chemotherapy 18, 798-806.

Anand, N. \& Davis, B. D. (1960). Damage by streptomycin to the cell membrane of Escherichia coli. Nature, London 185, 22-23.

ANand, N., Davis, B. D. \& ARmitage, A. K. (1960). Uptake of streptomycin by Escherichia coli. Nature, London 185, 23-24.

ANDRY, K. \& BOCKRATH, R. C. (1974). Dihydrostreptomycin accumulation in Escherichia coli. Nature, London 251, 534-536.

BACHMANN, B. J. \& Low, K. B. (1980). Linkage map of Escherichia coli $\mathrm{K}-12$, edition 6. Microbiological Reviews 44, 1-56.

Bryan, L. E. \& VAN Den Elzen, H. M. (1976). Streptomycin accumulation in susceptible and resistant strains of Escherichia coli and Pseudomonas aeruginosa. Antimicrobial Agents and Chemotherapy 9, 928-938.

Bryan, L. E. \& Van Den Elzen, H. M. (1977). Effects of membrane-energy mutations and cations on streptomycin and gentamicin accumulation by bacteria: a model for entry of streptomycin and gentamicin in susceptible and resistant bacteria. Antimicrobial Agents and Chemotherapy 12, 163-177.

Davis, B. D. \& Mingioli, E. S. (1950). Mutants of Escherichia coli requiring methionine or vitamin B12. Journal of Bacteriology 60, 17-28.

Demerec, M., Adelberg, E. A., Clark, A. J. \& Hartman, P. E. (1968). A proposal for a uniform nomenclature in bacterial genetics. Journal of General Microbiology 50, 1-14.

HANCOCK, R. (1960). The bactericidal action of streptomycin on Staphylococcus aureus and some accompanying biochemical changes. Journal of General Microbiology 23, 179-196.

HANCOCK, R. (1962a). Uptake of ${ }^{14}$ C-streptomycin by some micro-organisms and its relation to their streptomycin sensitivity. Journal of General Microbiology 28, 493-501.

HANCOCK, R. $(1962 b)$. Uptake of ${ }^{14}$ C-streptomycin by Bacillus megaterium. Journal of General Microbiology 28, 503-516.

HARVEY, R. J. (1973). Fraction of ribosomes synthesizing protein as a function of specific growth rate. Journal of Bacteriology 114, 287-293.

HöltJE, J.-V. (1978). Streptomycin uptake via an inducible polyamine transport system in Escherichia coli. European Journal of Biochemistry 86, 345-351.

HuRwitz, C. \& Rosano, C. L. (1962). Accumulation of label from $\mathrm{C}^{14}$-streptomycin by Escherichia coli. Journal of Bacteriology 83, 1193-1201.

КосH, A. L. (1980). The inefficiency of ribosomes functioning in Escherichia coli growing at moderate rates. Journal of General Microbiology 116, 165-171.

KOGUT, M. \& HaRRIS, M. (1969). Effects of streptomycin in bacterial cultures growing at different rates: interaction with bacterial ribosomes in vivo. European Journal of Biochemistry 9, 42-49.

MaAløe, O. \& KJELdGaARD, N. O. (1966). Control of Macromolecular Synthesis. New York, USA: Benjamin. 
Miller, M. H., Edberg, S. C., Mandel, L. J., Behar, C. F. \& Steigbigel, N. H. (1980). Gentamicin uptake in wild-type and aminoglycoside-resistant small-colony mutants of Staphylococcus aureus. Antimicrobial Agents and Chemotherapy 18, 722-729.

MUIR, M. E. \& WALLACE, B. J. (1979). Isolation of mutants of Escherichia coli uncoupled in oxidative phosphorylation using hypersensitivity to streptomycin. Biochimica et biophysica acta 547, 218-229.
Muir, M. E., Hanwell, D. R. \& Wallace, B. J. (1981). Characterization of a respiratory mutant of Escherichia coli with reduced uptake of aminoglycoside antibiotics. Biochimica et biophysica acta 638, 234-241.

TEMPEST, D. W. (1969). Quantitative relationships between inorganic cations and anionic polymers in growing bacteria. Symposia of the Society for General Microbiology 19, 87-111. 